\title{
Special fiber elements for thermal analysis of fiber-reinforced composites
}

\author{
Hui Wang \\ Institute of Scientific and Engineering Computation, \\ Henan University of Technology, Zhengzhou, China, and \\ Qinghua Qin \\ School of Engineering, Australian National University, Canberra, Australia
}

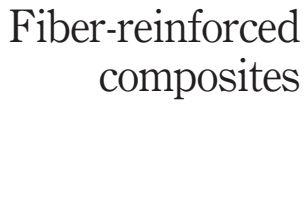

1079

\begin{abstract}
Purpose - The purpose of this paper is to present a new special element model for thermal analysis of composites.

Design/methodology/approach - A hybrid finite element formulation taking the fundamental solution as kernel function is presented in this work for analyzing the thermal behavior and predicting the effective thermal conductivity of fiber-reinforced composites. A representative volume cell containing single or multiple fibers (or inclusions) is considered to investigate the overall temperature distribution affected by the inclusions and the interactions among them, and to evaluate the effective thermal conductivity of the composites using the presented algorithm with special-purpose inclusion elements. Numerical examples are presented to demonstrate the accuracy and applicability of the proposed method in analyzing fiber-reinforced composites.

Findings - The independent intra-element field and frame field, as well as the newly-developed hybrid functional, make the algorithm versatile in terms of element construction, with the result that the related variational functional involves the element boundary integral only. All numerical results are compared with the solutions from ABAQUS and good agreement is observed for all cases, clearly demonstrating the potential applications of the proposed approach to large-scale modeling of fiber-reinforced composites. The usage of special inclusion element can significantly reduce model meshing effort and computing cost, and simultaneously avoid mesh regeneration when the fiber volume fraction is changed.

Practical implications - Due to the fact that the established special elements exactly satisfy the interaction of matrix and fiber within the element, only element boundary integrals are involved, thus the algorithm can significantly reduce modeling effort and computing cost with less elements, and simultaneously avoid mesh regeneration when the fiber volume fraction is changed.

Originality/value - Based on the special fundamental solution, a newly-constructed inclusion element is applied to a number of test problems involving unit RVCs with multiple fibers to access the accuracy of the model. The effective thermal conductivity of the composites is evaluated for cases of single and multiple fibers using the average temperatures at certain points on a data-collection surface. A new algorithm for evaluating effective properties with special elements is presented.
\end{abstract}

Keywords Programming and algorithm theory, Thermal properties of materials, Composite materials, Fiber-reinforced composites, Thermal analysis, Hybrid FEM, Fundamental solution,

Special inclusion element, Representative volume cell

Paper type Research paper

\section{Introduction}

Fiber-reinforced composites (Chung, 1994) are structural materials that usually consist of fiber reinforcing phase and matrix phase in which the fiber is embedded at a

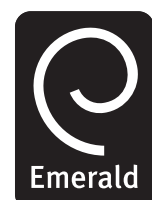

Engineering Computations: International Journal for Computer-Aided Engineering and

Vol. 28 No. 8,2011 pp. 1079-1097

(C) Emerald Group Publishing Limited 0264-4401 
EC 28,8

1080 macroscopic level to possibly experience a range of mechanical, thermal and chemical environment during their service life. The high thermal and electrical conductivity of fibers, as well as their high specific stiffness and high specific strength, make them useful as reinforcements for polymers, metals, carbons, and ceramics to increase the overall macroscopic modulus of the material. In past decades, therefore, fiber-reinforced composites have been widely used in engineering applications due to the superiority of their physical properties over the single matrix.

In fiber-reinforced composites, heat conduction behavior is an important and complex phenomenon and can usually be analyzed by micromechanical analysis using proper numerical methods, which must be capable of efficiently idealizing the individual fibers or bundle of fibers embedded within the matrix, and be sophisticated enough to take into account high-temperature gradients resulting from diffusion of temperature from the fiber to the matrix and allow for the interaction between fibers and the matrix.

Among numerical methods, the finite element method (FEM) (Chandrupatla and Belegundu, 2002) and boundary element method (BEM) (Brebbia et al., 1984; Qin, 1993) are often used for heat analysis in fiber-reinforced composites. The former is a domain-type approach and suitable for performing multi-domain analysis. However, the drawback of this method is that refined meshes are usually required around the fillers to achieve the desired accuracy. This is not practical for analysis of composites whose fiber distribution might change repeatedly. Unlike FEM, the BEM simply requires boundary division of the entire domain under consideration, reducing the dimensionality of the problem by one. Using this approach, steady-state and transient heat conduction (Ma et al., 2008), interface performance (Chen and Papathanasiou, 2004; Chen and Liu, 2001; Liu and Xu, 2000), and thermoelastic behavior (Banerjee and Henry, 1992; Henry et al., 2007; Liu et al., 2005; Wang and Yao, 2007) in fiber-reinforced composites have been investigated. However, singular or hyper-singular integrals are unavoidable in BEM. Furthermore, the BEM solution process becomes more complex when solving multi-material problems like fiber-reinforced composites, due to the requirement of continuity conditions across the interface between fiber and matrix. To overcome these difficulties, the hybrid Trefftz finite element (HT-FEM) was developed (Qin, 2000; Qin and Wang, 2008). This method is based on an independent intra-element field defined within the element, a frame field defined on the element boundary, and a hybrid variational functional. The HT-FEM approach finally yields a system involving boundary integrals only. Thus, it inherits the advantages of both conventional FEM and BEM, and has been successfully applied to various engineering problems (Jirousek and Qin, 1996; Qin, 1994, 1995, 2003, 2004).

As an alternative to HT-FEM, a new Green's function-based hybrid finite element formulation using a Green's functions or fundamental solutions instead of T-complete functions as kernel functions, named as HFS-FEM, was recently presented by Wang and Qin $(2009,2010)$ to retain the advantages of HT-FEM over FEM and BEM, and removes some drawbacks of it. The works in literatures (Wang and Qin, 2009, 2010) restrict to the isotropic heat transfer and orthotropic elasticity without local defects. However, HFS-FEM holds promise for solving problems with local effects. Thus, in this paper, formulations of HFS-FEM for the heat conduction analysis of fiber-reinforced composites is presented to simulate the effects of inclusions, based on a special fundamental solution which analytically satisfies the continuity of temperature and 
heat flux on the interface between fiber and matrix. Subsequently, a special purpose inclusion element is constructed for analyzing the interactions among fibers and interfacial heat effect in the composites. A representative volume cell (RVC) containing multiple fibers is constructed for estimating the effective thermal property of the composites. The accuracy of the numerical results obtained using the proposed method is assessed by comparing them with those obtained using ABAQUS, one of popular commercial softwares for finite element analysis. The comparison indicates that the proposed method is efficient and accurate for analyzing the thermal behavior of fiber-composites and has the potential to be scaled up to tackle large-scale practical problems of considerable interest.

\section{Statement of heat conduction problems}

\subsection{Mathematical model}

A two-dimensional mathematical model of steady-state heat conduction in the cross-section of the unidirectional fiber-reinforced composites is considered in this section. The fibers in the composites are assumed to be infinite parallel and have a reasonably circular shape with a fairly uniform diameter. For the sake of convenience, since matrix and fiber occupy different regions, the regions occupied by the isotropic matrix and fiber inclusions are referred to as regions $\Omega_{\mathrm{M}}$ and $\Omega_{\mathrm{F}}$, respectively, and the quantities associated with these regions are denoted by the corresponding subscripts M or F (Figure 1).

It is well known that an RVC for real composites with the smallest periodic repeat volume is usually selected to study the effective properties of composites in the micromechanics analysis (Figure 1). Without loss of generality, two-dimensional heat conduction problems in the square RVC with multiple fibers are considered, and the governing equations in terms of spatial variable $\mathbf{X}=\left(X_{1}, X_{2}\right)$ in matrix and fibers can, respectively, be written as:

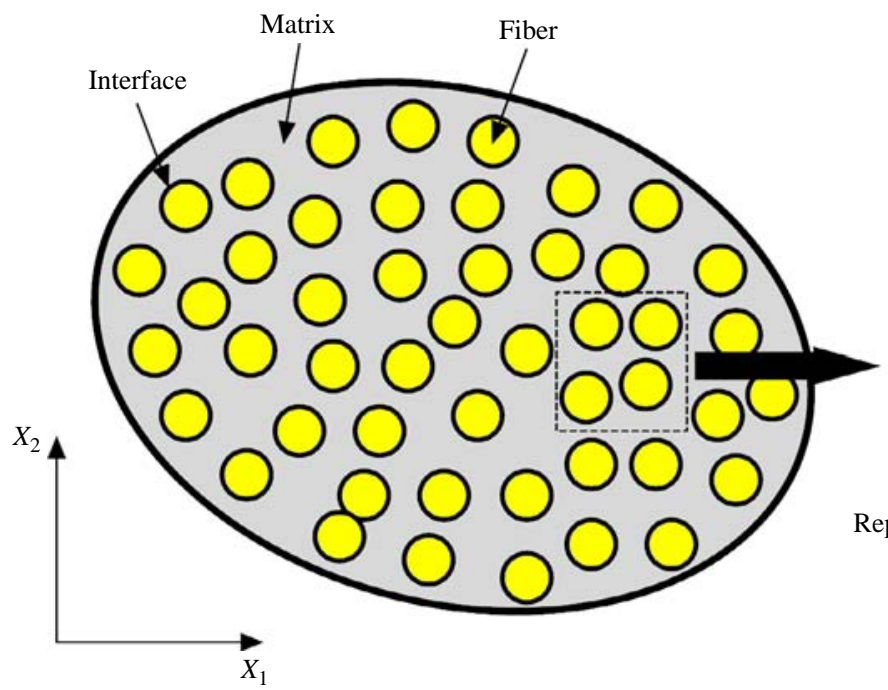

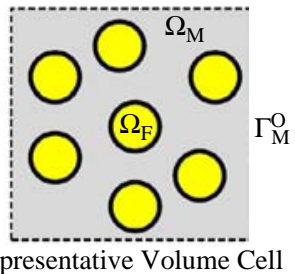

(RVC)
Fiber-reinforced composites

1081
Figure 1. Geometrical definition for plane heat conduction problems in fiber-reinforced composites 
EC

28,8

1082

$$
\begin{array}{ll}
k_{\mathrm{M}} u_{\mathrm{M}, i i}(\mathbf{X})=0 & \forall \mathbf{X} \in \Omega_{\mathrm{M}} \\
k_{\mathrm{F}} u_{\mathrm{F}, i i}(\mathbf{X})=0 & \forall \mathbf{X} \in \Omega_{\mathrm{F}}
\end{array}
$$

with the following boundary conditions applied on the outer boundary $\Gamma_{\mathrm{M}}^{\mathrm{O}}=$ $\Gamma_{u} \cup \Gamma_{q} \cup \Gamma_{c}$ of the matrix:

$$
\begin{cases}u_{\mathrm{M}}=\bar{u} & \text { on } \Gamma_{u} \\ q_{\mathrm{M}}=-k_{\mathrm{M}} u_{\mathrm{M}, i} n_{i}=\bar{q} & \text { on } \Gamma_{q} \\ q_{\mathrm{M}}=h_{e n v}\left(u_{\mathrm{M}}-u_{e n v}\right) & \text { on } \Gamma_{c}\end{cases}
$$

and the continuity conditions at the interface $\left(\Omega_{\mathrm{M}} \cap \Omega_{\mathrm{F}}\right)$ between the fiber and the matrix for the case of perfect bonding:

$$
\left\{\begin{array}{l}
u_{\mathrm{M}}=u_{\mathrm{F}} \\
q_{\mathrm{M}}+q_{\mathrm{F}}=0
\end{array}\right.
$$

where $u_{\mathrm{M}}$ and $u_{\mathrm{F}}$ are the temperature fields sought, $k_{\mathrm{M}}$ and $k_{\mathrm{F}}$ are the thermal conductivities and $n_{i}$ is the $i$ th component of the unit outward normal vector to the particular boundary. $q_{\mathrm{M}}$ and $q_{\mathrm{F}}$ represent the surface normal heat flux along the unit outward normal. $\bar{u}$ and $\bar{q}$ are specified functions on the corresponding boundaries. $h_{e m w}$ is the convection heat-transfer coefficient or film coefficient, and $u_{e n v}$ is the ambient environment temperature. The space derivatives are indicated by a comma, i.e. $u_{i}=\partial u / \partial X_{i}$, and the subscript index $i$ takes values 1 and 2 for the two-dimensional case. Additionally, the repeated subscript indices stand for the summation convention.

\subsection{Fundamental solutions}

Fundamental solutions play an important role in the derivation of the HFS-FEM formulation. The fundamental solution represents the material response at an arbitrary point when a unit point source is applied at a source point in an infinite domain. With the proposed HFS-FEM, for plane heat conduction problems in fiber-reinforced composites, two types of fundamental solution are used. One is the temperature response in an infinite matrix region $\Omega_{\mathrm{M}}(|z| \geq 0)$ in the absence of fibers (Figure 2(a)), and the other is the temperature response in an infinite matrix region $\Omega_{\mathrm{M}}(|z|>R)$ containing a circular fiber $\Omega_{\mathrm{F}}(|z|<R)$ (Figure 2(b)), where $z=x_{1}+x_{2} i$ is a complex number defined in a local coordinate system $\mathbf{x}=\left(x_{1}, x_{2}\right)$ with its origin coincident with the fiber center, and $i=\sqrt{-1}$ denotes the unit imaginary number.

(1) Fundamental solution without fiber. For the case of an infinite domain without fibres, assuming that a unit heat source is located at point $z_{0}$ in the infinite matrix domain $\Omega_{\mathrm{M}}$ (Figure 2(a)), the temperature response $G_{\mathrm{M}}$ at any field point $z$ is given in the form (Chao and Shen, 1997):

$$
G_{\mathrm{M}}\left(z, z_{0}\right)=-\frac{1}{2 \pi k_{\mathrm{M}}} \operatorname{Re}\left\{\ln \left(z-z_{0}\right)\right\}
$$

where Re denotes the real part of the bracketed expression. Clearly, the expression (4) shows singularity as $z=z_{0}$, which is the inherent feature of the fundamental solution. 


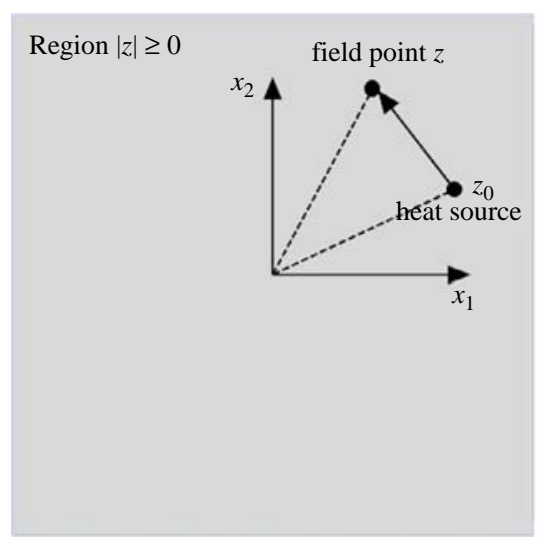

(a) Infinite domain without fiber

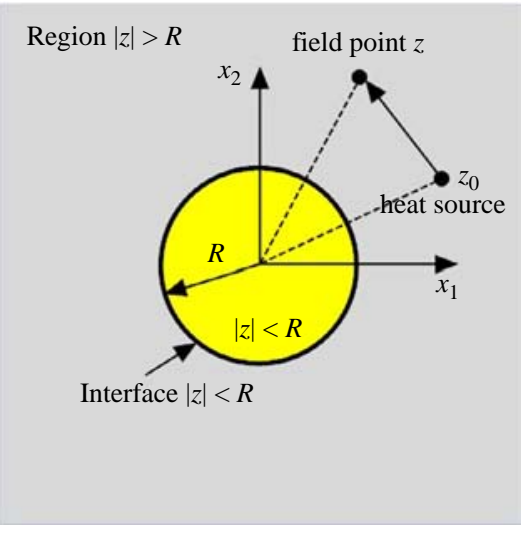

(b) Infinite domain with fiber

\section{Fiber-reinforced composites}

1083

Figure 2.

Fundamental solutions for plane heat conduction problems in fiber-reinforced composites

(2) Fundamental solution with fiber. For the case of an infinite domain with a centered circular fiber, consider a unit heat source located at the source point $z_{0}$ in the infinite matrix $\Omega_{\mathrm{M}}$ (Figure 2(b)). Then the temperature responses $G_{\mathrm{M}}$ and $G_{\mathrm{F}}$ at any field point $z$ in matrix and fiber regions are, respectively, obtained as (Chao and Shen, 1997):

$$
\begin{cases}G_{\mathrm{M}}=-\frac{1}{2 \pi k_{\mathrm{M}}}\left[\operatorname{Re}\left[\ln \left(z-z_{0}\right)\right]+\frac{k_{\mathrm{M}}-k_{\mathrm{F}}}{k_{\mathrm{M}}+k_{\mathrm{F}}} \operatorname{Re}\left[\ln \left(\frac{R^{2}}{z}-\bar{z}_{0}\right)\right]\right] & z \in \Omega_{\mathrm{M}} \\ G_{\mathrm{F}}=-\frac{1}{\left(k_{\mathrm{M}}+k_{\mathrm{F}}\right) \pi} \operatorname{Re}\left[\ln \left(z-z_{0}\right)\right] & z \in \Omega_{\mathrm{F}}\end{cases}
$$

using the complex potential theory and introducing the continuity condition (3) in the interface $|z|=R$. Similarly, the induced temperature $G_{\mathrm{M}}$ in the matrix shows a proper singular behavior at the source point $z_{0}$, while $G_{\mathrm{F}}$ in the fiber is regular because the source point $z_{0}$ is outside the fiber. Additionally, it is worth noting that since the fundamental solutions already include the presence of interface between the fillers and matrix, it is not necessary to model the temperature and heat flux continuity condition on the interface and then the analysis will become simpler. This is one of the advantages of the proposed approach stated below.

\section{The hybrid finite element formulation}

In this section, the formulation of the hybrid finite element model with fundamental solution as an interior trial function is presented for heat analysis of two-dimensional fiber-reinforced composites.

\subsection{Non-conforming intra-element field}

Applying the method of fundamental solution (Kupradze and Aleksidze, 1964) to remove the singularity of the fundamental solution, for a particular element, say element $e$, occupying a sub-domain $\Omega_{e}$ embedded with a centered circular fiber of radius $R$ and defined in a local reference system $\mathbf{x}=\left(x_{1}, x_{2}\right)$ whose axis remains parallel to the axis of the global reference system $\mathbf{X}=\left(X_{1}, X_{2}\right)$ (Figure 3 ), the temperature field at any 
$\mathrm{EC}$

28,8

\section{4}

\section{Figure 3.}

Intra-element field, frame field in a particular element in HFS-FEM, and the generation of source points for a particular inclusion element

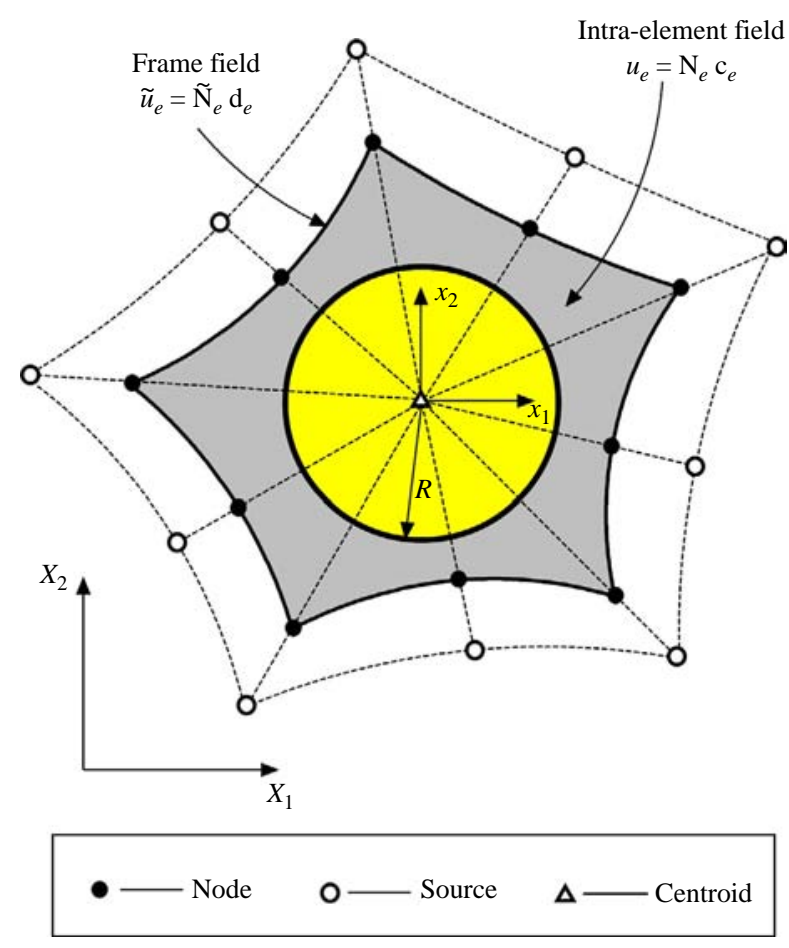

point $\mathbf{x}$ within the element domain is assumed to be a linear combination of fundamental solutions centered at different source points $\mathbf{x}_{s j}$, that is:

$$
u_{e}(\mathbf{x})=\sum_{j=1}^{n_{s}} G_{e}\left(\mathbf{x}, \mathbf{x}_{s j}\right) c_{e j}=\mathbf{N}_{e}(\mathbf{x}) \mathbf{c}_{e} \quad \forall \mathbf{x} \in \Omega_{e}, \mathbf{x}_{s j} \notin \Omega_{e}
$$

where $c_{e j}$ represents undetermined coefficients, $n_{s}$ is the number of virtual sources outside the element $e$, and $G_{e}\left(\mathbf{x}, \mathbf{x}_{s j}\right)$ represents the corresponding fundamental solution, which can be conveniently expressed using a unified form:

$$
G_{e}\left(\mathbf{x}, \mathbf{x}_{s j}\right)= \begin{cases}G_{\mathrm{M}}\left(\mathbf{x}, \mathbf{x}_{s j}\right) & \mathbf{x} \in \Omega_{\mathrm{M}} \\ G_{\mathrm{F}}\left(\mathbf{x}, \mathbf{x}_{s j}\right) & \mathbf{x} \in \Omega_{\mathrm{F}}\end{cases}
$$

In practice, the location of sources affects the final accuracy (Mitic and Rashed, 2004; Wang and Qin, 2007, 2008; Wang et al., 2006) and can usually be determined by means of the formulation (Young et al., 2006):

$$
\mathbf{x}_{s}=\mathbf{x}_{b}+\gamma\left(\mathbf{x}_{b}-\mathbf{x}_{c}\right)
$$

where $\gamma$ is a dimensionless coefficient, $\mathbf{x}_{b}$ is the elementary boundary point and $\mathbf{x}_{c}$ the geometrical centroid of the element. For a particular element as shown in Figure 3, we can use the nodes of element to generate related source points using the relation (8). 
The corresponding outward normal derivative of $u_{e}$ on $\Gamma_{e}$ is defined by:

$$
q_{e}=-k_{M} \frac{\partial u_{e}}{\partial n}=\mathbf{Q}_{e} \mathbf{c}_{e}
$$

where:

$$
\mathbf{Q}_{e}=-k_{M} \frac{\partial \mathbf{N}_{e}}{\partial n}=-k_{M} \mathbf{A} \mathbf{T}_{e}
$$

with:

$$
\mathbf{A}=\left[\begin{array}{ll}
n_{1} & n_{2}
\end{array}\right], \quad \mathbf{T}_{e}=\left[\begin{array}{ll}
\frac{\partial \mathbf{N}_{e}}{\partial x_{1}} & \frac{\partial \mathbf{N}_{e}}{\partial x_{2}}
\end{array}\right]^{\mathrm{T}}
$$

\subsection{Auxiliary conforming frame field}

In order to enforce conformity on the field variable $u$, for instance, $u_{e}=u_{f}$ on $\Gamma_{e} \cap \Gamma_{f}$ of any two neighboring elements $e$ and $f$, an auxiliary inter-element frame field $\tilde{u}$ independent of the intra-element field is introduced in terms of the same nodal degrees of freedom (DOF), $\mathbf{d}$, as used in conventional FEMs. In this case, $\tilde{u}$ is confined to the whole element boundary, that is:

$$
\tilde{u}_{e}(\mathbf{x})=\tilde{\mathbf{N}}_{e}(\mathbf{x}) \mathbf{d}_{e}
$$

where $\tilde{\mathbf{N}}_{e}$ represents the conventional finite element interpolating functions. For example, a simple interpolation of the frame field on any side of a particular element (Figure 4) can be given in the form:

$$
\tilde{u}=\tilde{N}_{1} u_{1}+\tilde{N}_{2} u_{2}+\tilde{N}_{3} u_{3}
$$

where $\tilde{N}_{i}(i=1,2,3)$ stands for shape functions in terms of natural coordinate $\xi$ shown in Figure 4.

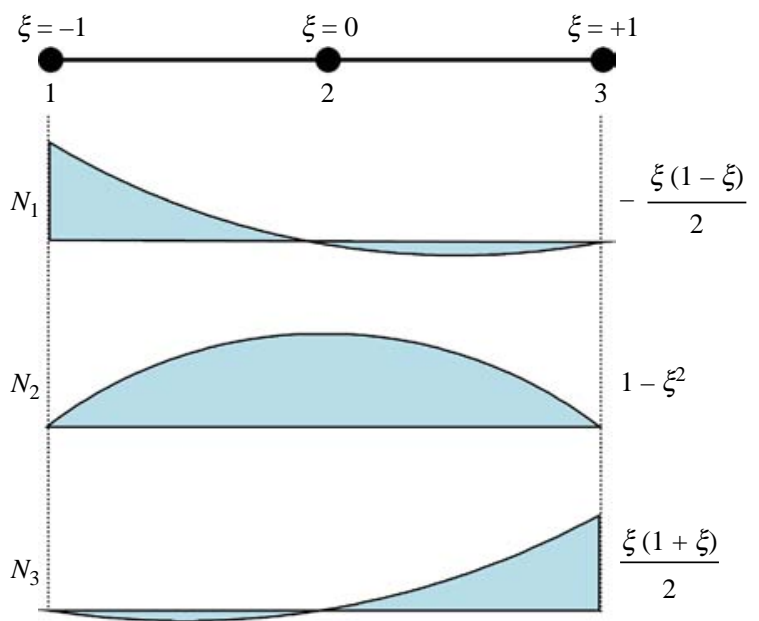

Figure 4. Typical quadratic interpolation for the frame field 
EC

28,8

1086

\subsection{Modified variational principle and stiffness equation}

For the boundary value problem defined in equations (1) and (2), since the stationary conditions of the traditional potential or complementary variational functional cannot guarantee the satisfaction of the continuity condition on the inter-element boundary, which is required in the proposed hybrid finite element model, a modified potential functional (Wang and Qin, 2009) is developed as follows:

$$
\Pi_{m}=\sum_{e} \Pi_{m e}
$$

with:

$$
\Pi_{m e}=-\frac{1}{2} \int_{\Omega_{e}} k u_{, i} u_{, i} \mathrm{~d} \Omega-\int_{\Gamma_{q e}} \bar{q} \tilde{u} \mathrm{~d} \Gamma+\int_{\Gamma_{e}} q(\tilde{u}-u) \mathrm{d} \Gamma
$$

in which the governing equation (1) is assumed to be satisfied, a priori, due to the use of the fundamental solution in the HFS FE model. The boundary $\Gamma_{e}$ of a particular element consists of the following parts:

$$
\Gamma_{e}=\Gamma_{u e} \cup \Gamma_{q e} \cup \Gamma_{I e}
$$

where $\Gamma_{I e}$ represents the inter-element boundary of the element " $e$ ".

Appling the divergence theorem:

$$
\int_{\Omega} f_{, i} h_{, i} \mathrm{~d} \Omega=\int_{\Gamma} h f_{, i} n_{i} \mathrm{~d} \Gamma-\int_{\Omega} h \nabla^{2} f \mathrm{~d} \Omega
$$

for any smooth functions $f$ and $h$ in the domain, we can eliminate the domain integral from equation (15) and obtain following functional for the HFS FE model:

$$
\Pi_{m e}=-\frac{1}{2} \int_{\Gamma_{e}} q u \mathrm{~d} \Gamma-\int_{\Gamma_{q e}} \bar{q} \tilde{u} \mathrm{~d} \Gamma+\int_{\Gamma_{e}} q \tilde{u} \mathrm{~d} \Gamma
$$

Then, substituting equations (6), (9) and (12) into the functional equation (18) produces:

$$
\Pi_{e}=-\frac{1}{2} \mathbf{c}_{e}^{\mathrm{T}} \mathbf{H}_{e} \mathbf{c}_{e}-\mathbf{d}_{e}^{\mathrm{T}} \mathbf{g}_{e}+\mathbf{c}_{e}^{\mathrm{T}} \mathbf{G}_{e} \mathbf{d}_{e}
$$

in which:

$$
\mathbf{H}_{e}=\int_{\Gamma_{e}} \mathbf{Q}_{e}^{\mathrm{T}} \mathbf{N}_{e} \mathrm{~d} \Gamma \quad \mathbf{G}_{e}=\int_{\Gamma_{e}} \mathbf{Q}_{e}^{\mathrm{T}} \tilde{\mathbf{N}}_{e} \mathrm{~d} \Gamma \quad \mathbf{g}_{e}=\int_{\Gamma_{e q}} \tilde{\mathbf{N}}_{e}^{\mathrm{T}} \bar{q} \mathrm{~d} \Gamma
$$

To enforce inter-element continuity on the common element boundary, the unknown vector $\mathbf{c}_{e}$ should be expressed in terms of nodal DOF $\mathbf{d}_{e}$ using the variational approach. Minimization of the functional $\Pi_{e}$ with respect to $\mathbf{c}_{e}$ and $\mathbf{d}_{e}$, respectively, yields:

$$
\frac{\partial \Pi_{e}}{\partial \mathbf{c}_{e}^{\mathrm{T}}}=-\mathbf{H}_{e} \mathbf{c}_{e}+\mathbf{G}_{e} \mathbf{d}_{e}=\mathbf{0} \quad \frac{\partial \Pi_{e}}{\partial \mathbf{d}_{e}^{\mathrm{T}}}=\mathbf{G}_{e}^{\mathrm{T}} \mathbf{c}_{e}-\mathbf{g}_{e}=\mathbf{0}
$$

from which the optional relationship between $\mathbf{c}_{e}$ and $\mathbf{d}_{e}$, and the stiffness equation can be obtained: 


$$
\mathbf{K}_{e} \mathbf{d}_{e}=\mathbf{g}_{e} \quad \mathbf{c}_{e}=\mathbf{H}_{e}^{-1} \mathbf{G}_{e} \mathbf{d}_{e}
$$

where $\mathbf{K}_{e}=\mathbf{G}_{e}^{\mathbf{T}} \mathbf{H}_{e}^{-1} \mathbf{G}_{e}$ stands for the element stiffness matrix.

It is worth noting that the evaluation of the right-hand vector $\mathbf{g}_{e}$ in equation (21) is the same as that in conventional FEM, which is obviously convenient for the implementation of HFS-FEM into existing FEM programs.

\section{Four types of elements in the proposed HFS-FEM}

One of the advantages of the HFS-FEM procedure described above is that the element used in HFS-FEM can be arbitrarily constructed to adapt to different geometries of the problem. In this work, for the sake of convenience, we restrict our analysis to the four types of elements shown in Table I, from which we can see that GE1 and GE2 are general elements suitable for a region without inclusions, and SE1 and SE2 are delicate special

\begin{tabular}{ll}
\hline Name & Description \\
\hline
\end{tabular}

GE1 General eight-node quadrilateral element used for region without inclusions

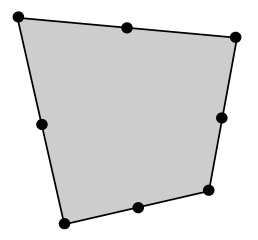

GE2 General 16-node quadrilateral element used for region without inclusions

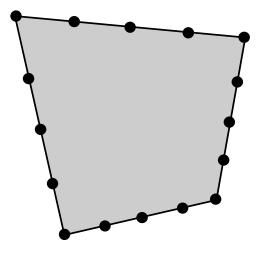

SE1 Special-purpose eight-node quadrilateral circular inclusion element used for region with fibers

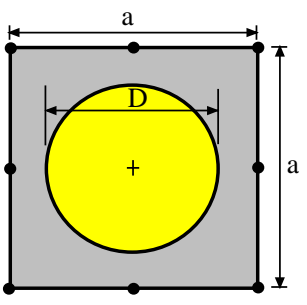

SE2 Special-purpose 16-node quadrilateral circular inclusion element used for region with fibers

\section{Fiber-reinforced composites}

1087 
EC

28,8

\section{8}

inclusion elements with different nodes suitable for fiber analysis. Owing to the geometrical symmetry of the circular fiber, the special element is designed as a square with side length $a$. If the diameter of the fiber in it is denoted as $D$, the parameter $D / a$, also termed the aspect ratio in this work, is introduced to determine the distance between the element boundary and the inclusion boundary. Also, the variation of parameter $D / a$ corresponds to the change of fiber volume fraction.

\section{Effective thermal conductivity}

The effective thermal conductivity is a very important parameter for engineering applications of composites. Usually the RVC approach is utilized in micro-mechanical model development. In this paper, a general square RVC with random multiple inclusions is used to investigate the effect of fiber size and to evaluate the effective thermal properties for the case of two-dimensional heat conduction (Figure 5(a)). The side length of the RVC is taken to be $L$. Meanwhile, an effective homogeneous model with the same geometry as the RVC is assumed with constant effective thermal conductivity $k_{C}$.

According to Fourier's law, the thermal conductivity along the $i-$ direction is defined as:

$$
k_{i}=-\frac{q_{i}}{\left(\partial u / \partial x_{i}\right)}
$$

Therefore, the effective thermal conductivity of the equivalent homogeneous model (Figure 5(b)) can be computed by applying appropriate boundary conditions. For example, in the homogeneous model, if:

- a uniform heat flux $\mathrm{f}>q_{0}$ is horizontally applied on the left side of the square;

- the temperature on the right side remains zero; and

- both the top and bottom sides are insulated, then, the temperature distribution in the model is linear in the horizontal direction; and the heat flux component in the

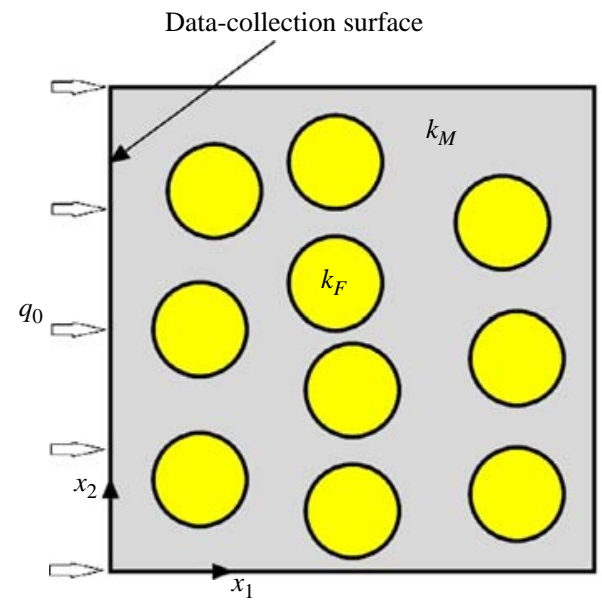

(a) Micro-mechanical model (RVC)

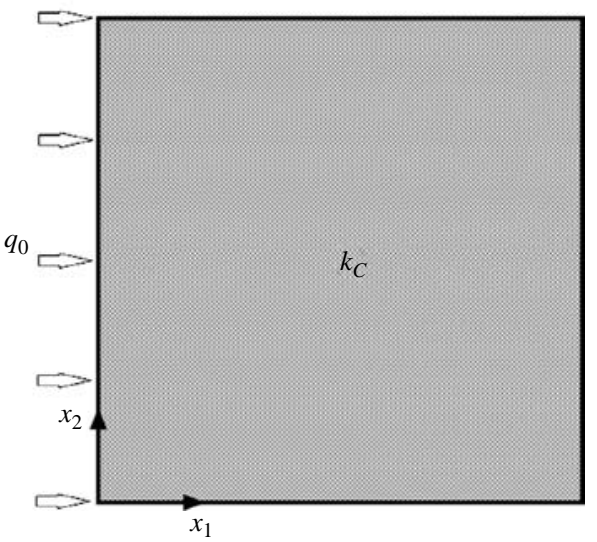

(a) Effective homogeneous model
Figure 5.

Micro-mechanical model (RVC) and effective homogeneous model 
body is constant, subsequently, the effective thermal conductivity $k_{c}$ in the horizontal direction can be evaluated by the following formula:

$$
k_{C}=-\frac{q_{0}}{\left(\partial u / \partial x_{1}\right)}=-\frac{q_{0}}{\left(\Delta u / \Delta x_{1}\right)}=-\frac{q_{0}}{\left(u_{\text {left }} / L\right)}
$$

where $\Delta u$ is the temperature difference between the left and right surfaces and $u_{\text {left }}$ represents the temperature on the left surface.

On the basis of the above discussion, the effective thermal conductivity can be estimated from the real RVC with multiple fibers by applying the same boundary conditions as those applied in the effective model, and using the temperature results on the left and right, two data-collection sides, that is:

$$
k_{C}=-\frac{q_{0}}{\left(\tilde{u}_{\text {left }} / L\right)}
$$

where $\tilde{u}_{\text {left }}$ is the average temperature on the left data-collection surface, which can be evaluated from nodal temperatures obtained by the presented hybrid finite element formulation.

\section{Numerical accuracy}

To demonstrate the accuracy of the presented hybrid algorithm and to investigate the thermal response induced by the fibers, RVCs with one, five and nine fibers are discussed in this section. Then the effective thermal properties are evaluated for the three cases. All results are compared with numerical solutions from ABAQUS for verifications.

\subsection{Unit square RVC with single fiber}

To access the accuracy of the present hybrid finite element formulation with special purpose inclusion elements, as the first verification example, the thermal behavior in a unit square RVC with a centered circular fiber is studied. In the calculation, a specified uniform heat flux $q_{0}$ with value of -100 is considered. Moreover, the thermal conductivity in units consistent with that of $q_{0}$ is assumed to be $k_{\mathrm{M}}=1$ and $k_{\mathrm{F}}=20$ for the matrix and the fiber, respectively.

To study the effect of location of source points which are outside the element, let the parameter $\gamma$ vary from 0 to 10 . In the analysis, the domain is modeled with only one special element (Figure 6(b) and (c)) and the radius of the fiber is assumed to be 0.1. The variation of temperature at the central point of the fiber is shown in Figure 7 as a function of the parameter $\gamma$. The solution presented is compared with that from ABAQUS and is found to be in good agreement; however, ABAQUS used 828 elements (Figure 6(a)). It is also evident from Figure 7 that a stable result can be obtained using the proposed hybrid special elements when the value of $\gamma$ is within the interval $(1,7)$. Meanwhile, we also found that, for the special elements both SE1 and SE2, the use of too small or too large a value of the parameter $\gamma$ results in low accuracy and an unstable solution, due to the potential singularity disturbance of the fundamental solution when the source points are close to the physical element boundary or to the possible round-off error in floating point arithmetic (Mitic and Rashed, 2004; Wang et al., 2006). Thus, in the following computation, $\lambda=4.0$ is used.

\section{Fiber-reinforced composites}

1089 
EC

28,8

\section{0}

Figure 6.

Mesh division of ABAQUS and HFS-FEM

for the case of single fiber

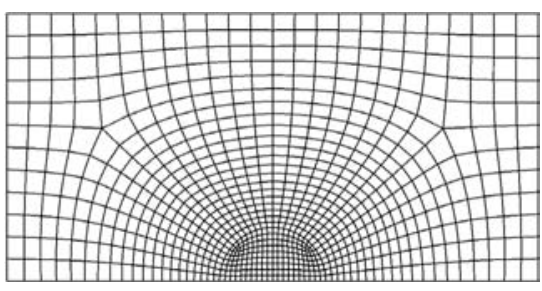

(a) ABAQUS mesh for half model

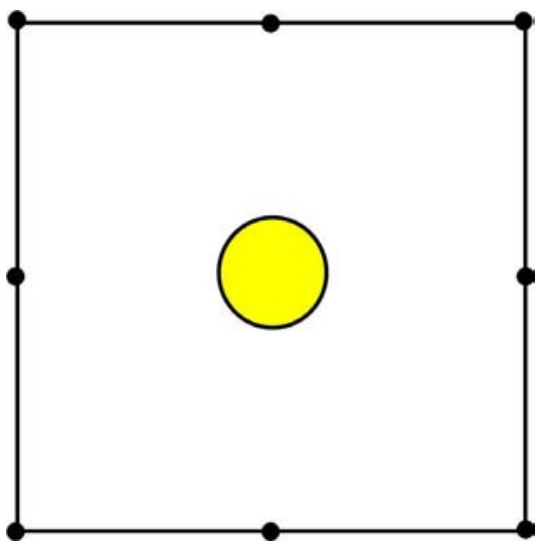

(b) HFS-FEM mesh with 8 nodes

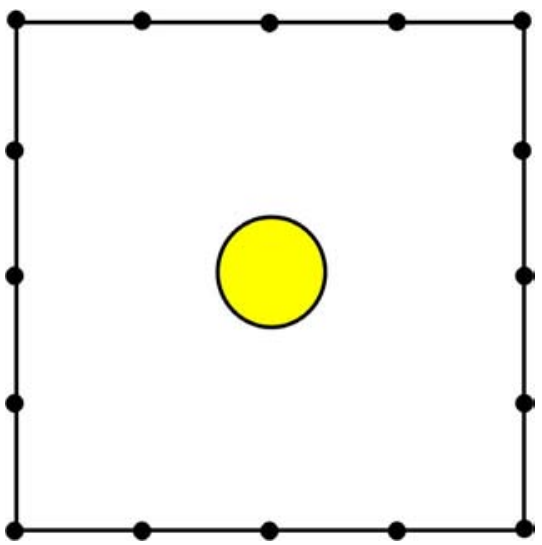

(c) HFS-FEM mesh with 16 nodes

To demonstrate the efficiency of the constructed circular fiber element, and to investigate the effect of fiber size on temperature variation, the aspect ratio $D / a$ is designed to vary from 0 to 1 . The corresponding variations of temperature and heat flux at the central point of the fiber are shown in Figures 8 and 9, respectively. Compared to the results from ABAQUS, which are achieved by refined meshes around and within the 


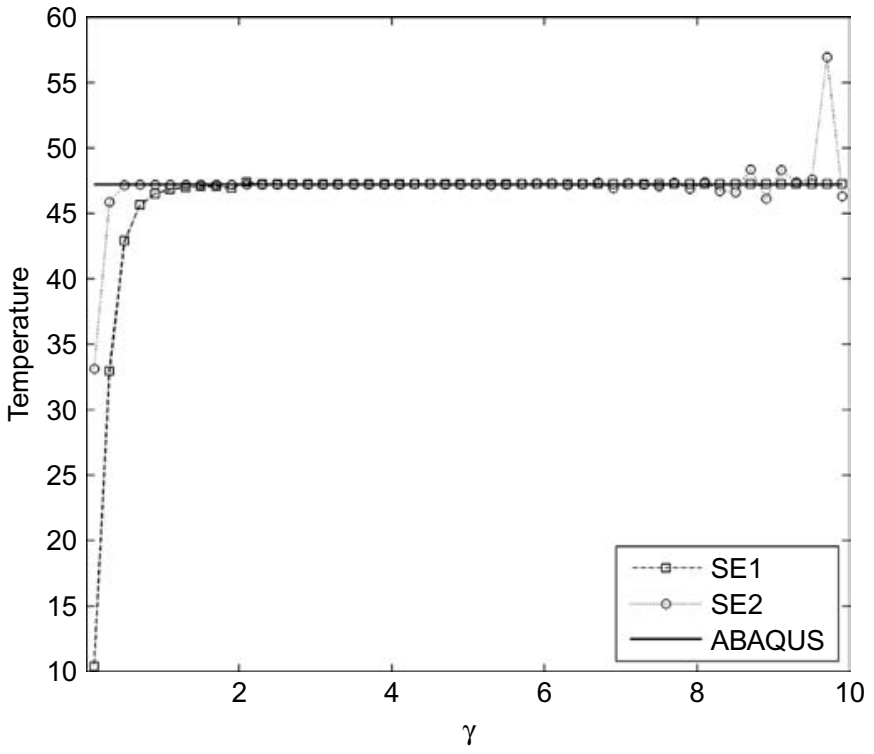

\section{Fiber-reinforced composites}

1091

Figure 7.

Temperature variation at the central point of the fiber with different parameter $\gamma$

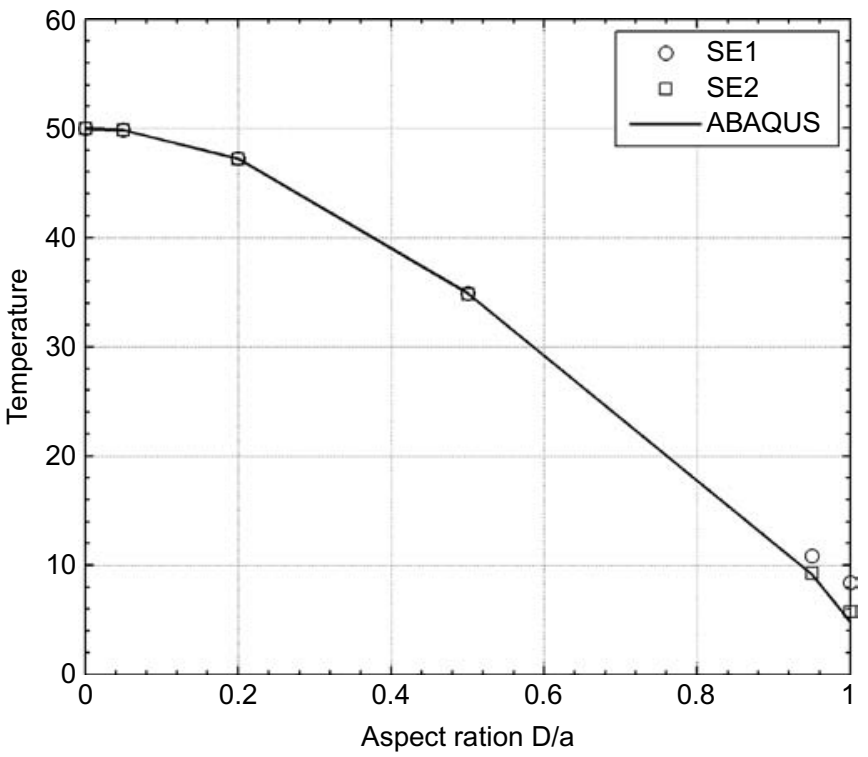

Figure 8.

Effect of aspect ratio of element and fiber on the temperature at the central point of the fiber

fiber in a half model by virtue of symmetry, the special purpose inclusion elements achieve similar accuracy but with one element only. More importantly, it can be seen that the increase of aspect ratio corresponding to the increase of fiber volume fraction in the element does not require the mesh regeneration in the presented approach with special fiber element, as expected. A slight deviation close to the largest aspect ratio as observed from the plot (Figure 8) may be attributed to the fact that elements with few 
EC

28,8

\section{2}

Figure 9.

Effect of aspect ratio of element and fiber on the heat flux at the central point of the fiber

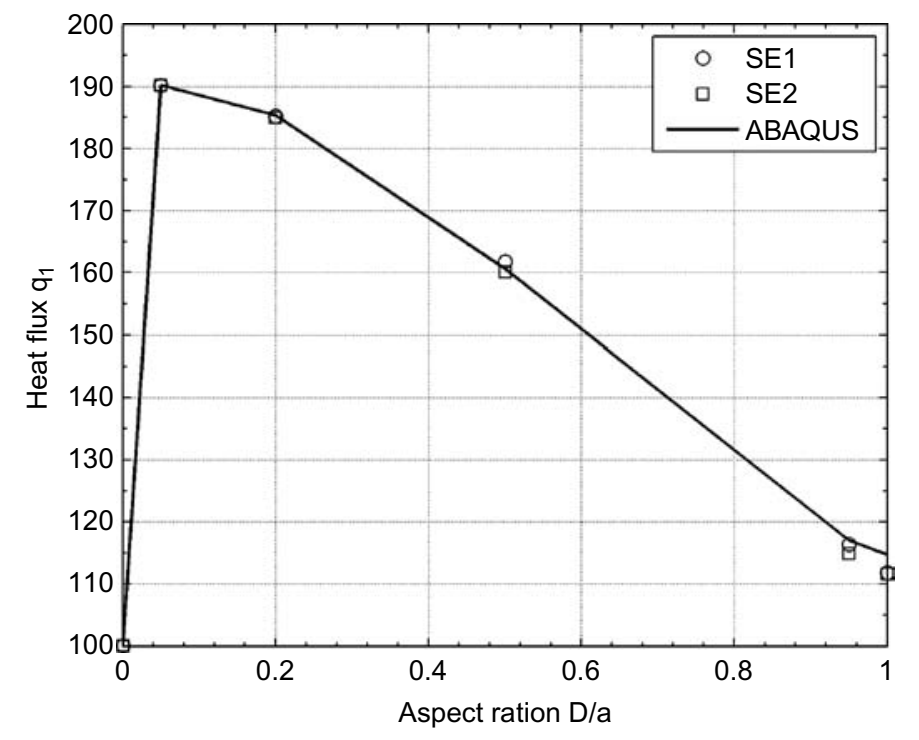

nodes cannot fully capture the strong interaction of the fiber and the physical boundary, so the special element SE2 with 16 nodes are recommended for the following computation. Simultaneously, we see that the maximum relative error of heat flux in Figure 9 is less 4 percent when just one special element is used. From these two figures we can also see that as the aspect ratio increases, which means that the element boundary is close to the interface of the fiber, the sought temperature tends to decrease. The reason is that the larger diameter of the fiber implies a larger volume fraction of the fiber, so the overall conductivity of the RVC under consideration is increased. Hence, the heat from the applied flux is carried away to the face, which is maintained at $0^{\circ} \mathrm{C}$ at a higher rate than in the case of pure matrix without fibers.

Figure 10 shows the temperature variation along the line $X_{2}=0.5$ when the radius of the fiber is maintained at 0.1 . As the interface of fiber and matrix plays an important role in heat conduction from matrix region to fiber region, the interfacial temperature is shown in Figure 11. Good agreement between HFS-FEM and ABAQUS is again observed. Furthermore, we can see that the existence of the fiber strongly affects the temperature distribution, especially in the region near the fiber and on the surface, to which the uniform heat flux is applied, compared to the temperature without fiber. The lower surface temperature of the composite is useful for the engineering applications. We also note that the temperature changes around the fiber are small, the reason being that the thermal conductivity of the fiber is usually several ten times that of the matrix, so the heat can flow through the fiber rapidly.

\subsection{Unit square RVC with multiple fibers}

Next we examine the heat conduction in unit square RVCs with five and nine fibers (Figure 12). As before, a uniform flux of 100.0 is applied to the left side of the RVC. The thermal conductivity of the fiber is again assumed to be 20 times that of the matrix and the radius of the fiber to be 0.1 . 


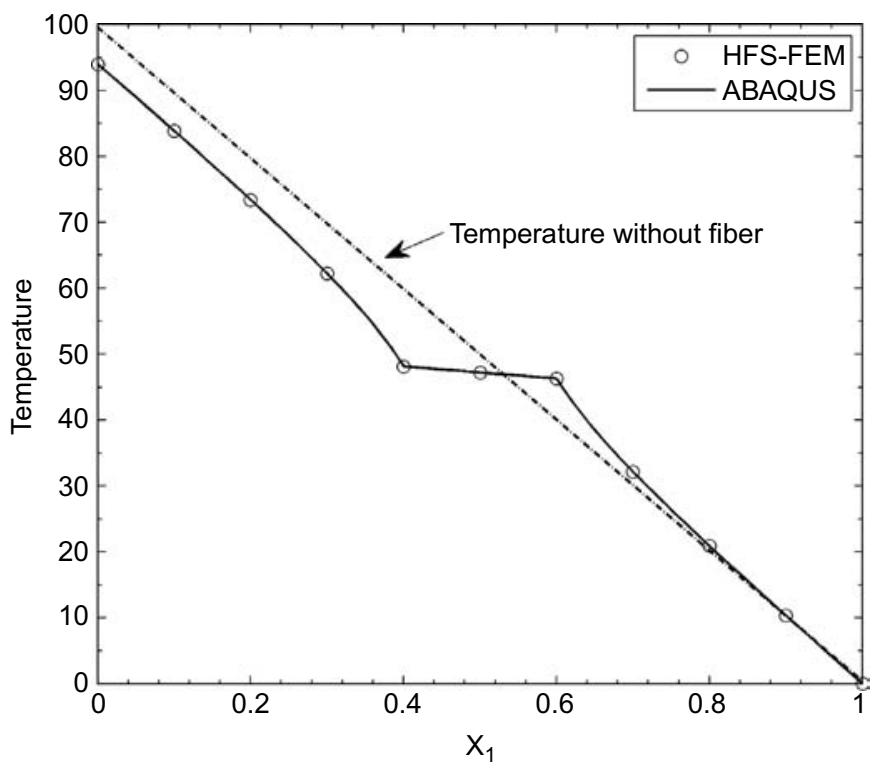

\section{Fiber-reinforced composites}

1093

Figure 10.

Temperature variation along the line $X_{2}=0.5$

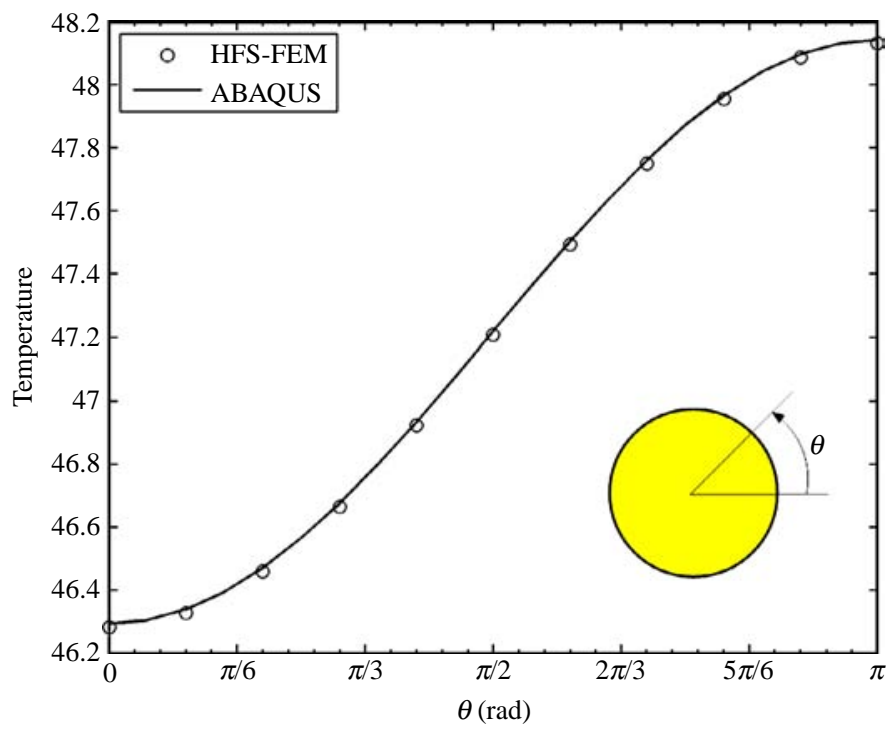

Figure 11. Distribution of temperature along the boundary of the fiber

The HFS-FEM meshes for computation of the RVCs with five and nine fibers are shown in Figure 13, in which five and nine SE1 elements are used for the cases of Figure 12(a) and (b), respectively. Figure 14 shows the distribution of temperature field along the mid-line $X_{2}=0.5$. It can be seen from Figure 14 that the HFS-FEM results are in good agreement with the corresponding finite element solutions using ABAQUS, even though many fewer elements are used in the proposed model. As expected, 
EC

28,8

\section{4}

Figure 12.

RVCs with multiple fiber inclusions

Figure 13.

HFS-FEM mesh division for RVC with five (left) and nine (right) fibers

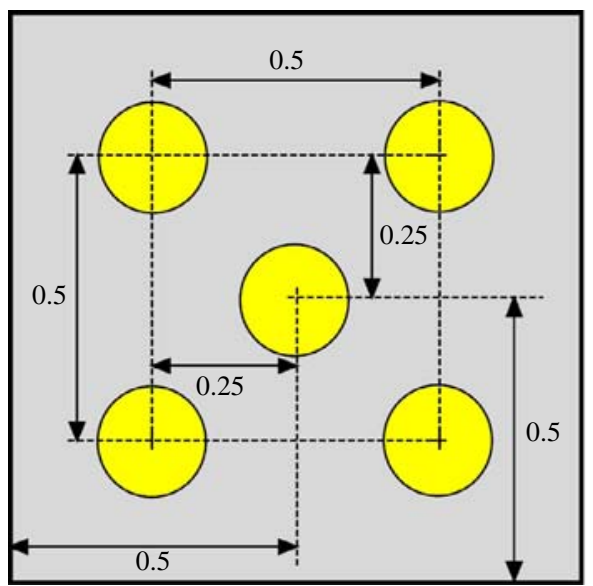

(a) zig-zag mode with five fibers
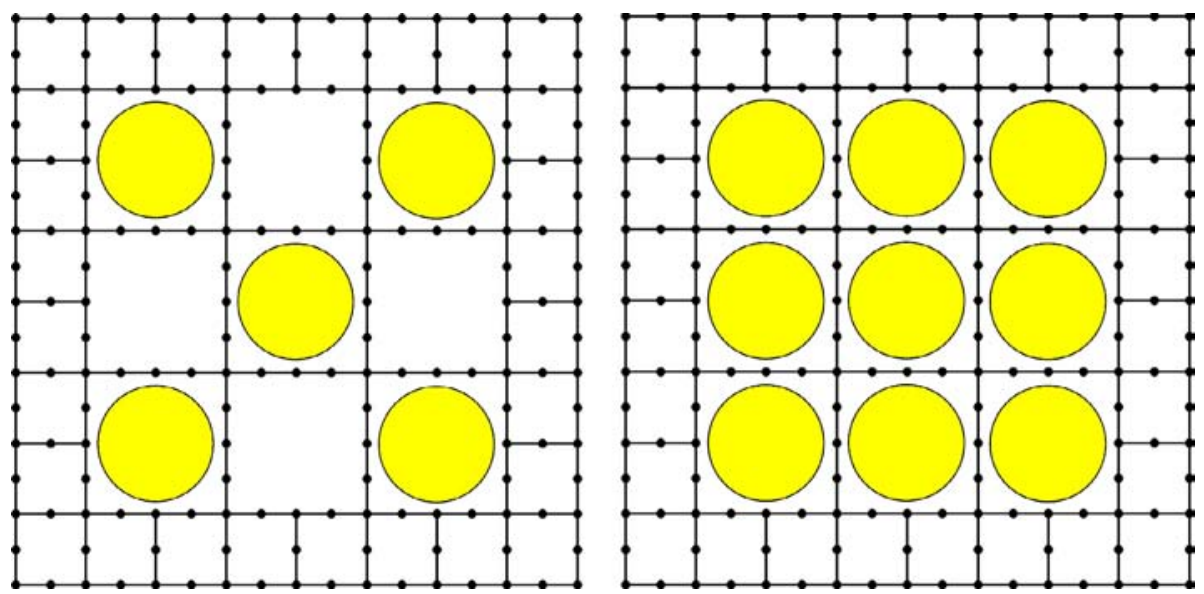

quasi-step curves are also evident in the temperature field near the fibers, and the surface temperature of the body displays a clear decrease as the number of fibers increases. For example, the temperature at the mid-point on the data-collection surface of the body changes from $100^{\circ} \mathrm{C}$ (without fiber) to $93.74^{\circ} \mathrm{C}$ (single fiber), to $75.66^{\circ} \mathrm{C}$ (five fibers), and finally to $57.37^{\circ} \mathrm{C}$ (nine fibers). Moreover, comparing the temperature over the mid-line and the average temperature shown in Table II we can conclude that the temperature evidences a weak change on the data-collection surface. The temperature distribution in the entire domain is shown in Figure 15, showing clearly the variation of temperature due to the presence of fibers.

Finally, the effective thermal conductivity of the fiber-reinforced composites along the $x_{1}$-direction is estimated using formula (24). To this end, the average temperature at all nodes on the data-collection surface is first evaluated and listed in Table II; then, the desired effective thermal conductivity is obtained by dividing the specified uniform 

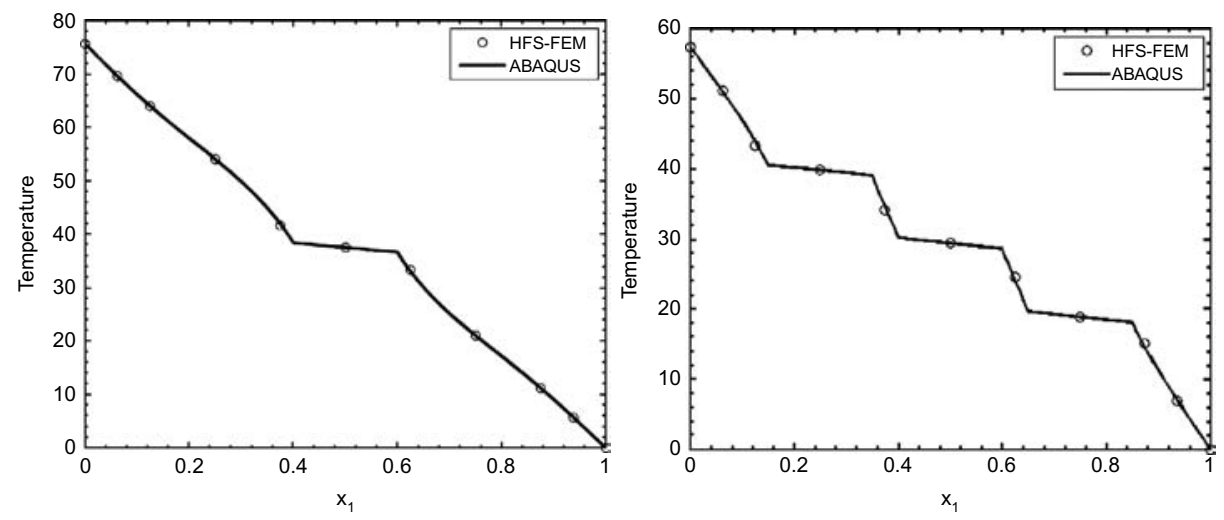

Fiber-reinforced
composites

1095

Figure 14.

Temperature variation along the line $X_{2}=0.5$ for

the cases of five fibers (left) and nine fibers (right)

\begin{tabular}{lllll}
\hline & & One fiber & Five fibers & \multicolumn{1}{c}{ Nine fibers } \\
\hline Average temperature on the & HFS-FEM & $94.541(16)^{\mathrm{a}}$ & $75.125(180)$ & $59.136(180)$ \\
data-collection surface & ABAQUS & $94.479(2,593)$ & $75.105(5,265)$ & $58.946(11,557)$ \\
Effective thermal conductivity & HFS-FEM & 1.0577 & 1.3311 & 1.6910 \\
& ABAQUS & 1.0584 & 1.3315 & 1.6965
\end{tabular}

Note: ${ }^{\mathrm{a} D a t a}$ in bracket represent the DOF used in the computation

Table II.

Average temperature on the data-collection surface and effective thermal conductivity of composites
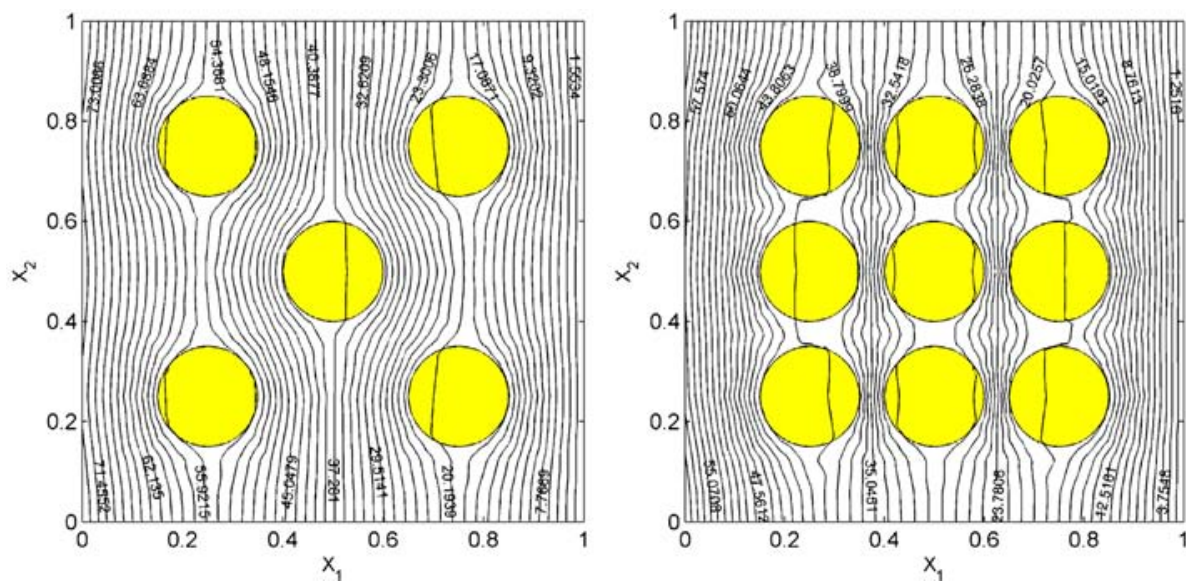

Figure 15. Contour of temperature in the RVCs under consideration with five fibers (left) and nine fibers (right)

heat flux by the obtained average temperature. The results obtained from the HFS-FEM are listed in Table II and compared with those from ABAQUS. Excellent agreement is observed, illustrating the efficiency and accuracy of the proposed hybrid finite element formulation. 
EC

28,8

1096

\section{Conclusions}

A new hybrid finite element model suitable for application to large-scale RVC analysis of heat conduction problems in fiber-reinforced composites is presented. The independent intra-element field and frame field, as well as the newly developed hybrid functional, make the algorithm versatile in terms of element construction, with the result that the related variational functional involves the element boundary integral only. Based on the special fundamental solution, a newly constructed inclusion element is applied to a number of test problems involving unit RVCs with multiple fibers to access the accuracy of the model. The effective thermal conductivity of the composites is evaluated for cases of single and multiple fibers using the average temperatures at certain points on a data-collection surface. All numerical results are compared with the solutions from ABAQUS and good agreement is observed for all cases, clearly demonstrating the potential applications of the proposed approach to large-scale modeling of fiber-reinforced composites. The usage of special inclusion element can significantly reduce model meshing effort and computing cost, and simultaneously avoid mesh regeneration when the fiber volume fraction is changed.

\section{References}

Banerjee, P.K. and Henry, D.P. (1992), "Elastic analysis of three-dimensional solids with fiber inclusions by BEM", International Journal of Solids and Structures, Vol. 29, pp. 2423-40.

Brebbia, C.A., Telles, J.C.F. and Wrobel, L.C. (1984), Boundary Element Techniques, Springer, Berlin.

Chandrupatla, T.R. and Belegundu, A.D. (2002), Introduction to Finite Elements in Engineering, 3rd ed., Prentice-Hall, Upper Saddle River, NJ.

Chao, C.K. and Shen, M.H. (1997), "On bonded circular inclusions in plane thermoelasticity", Journal of Applied Mechanics, Vol. 64, pp. 1000-4.

Chen, X. and Papathanasiou, T.D. (2004), "Interface stress distributions in transversely loaded continuous fiber composites: parallel computation in multi-fiber RVEs using the boundary element method", Composites Science and Technology, Vol. 64, pp. 1101-14.

Chen, X.L. and Liu, Y.J. (2001), "Multiple-cell modeling of fiber-reinforced composites with the presence of interphases using the boundary element method", Computational Materials Science, Vol. 21, pp. 86-94.

Chung, D.D.L. (1994), Carbon Fiber Composites, Butterworth-Heinemann, Boston, MA.

Henry, D.P., Ma, F., Chatterjee, J. and Banerjee, P.K. (2007), "Steady state thermoelastic analysis of 3D solids with fiber inclusions by boundary element method", Computer Methods in Applied Mechanics and Engineering, Vol. 197, pp. 294-307.

Jirousek, J. and Qin, Q.H. (1996), “Application of hybrid-Trefftz element approach to transient heat conduction analysis", Computers \& Structures, Vol. 58, pp. 195-201.

Kupradze, V.D. and Aleksidze, M.A. (1964), "The method of functional equations for the approximate solution of certain boundary value problems", USSR Comput. Math. Math. Phys., Vol. 4, pp. 82-126.

Liu, Y.J. and Xu, N. (2000), "Modeling of interface cracks in fiber-reinforced composites with the presence of interphases using the boundary element method", Mechanics of Materials, Vol. 32, pp. 769-83.

Liu, Y.J., Nishimura, N., Otani, Y., Takahashi, T., Chen, X.L. and Munakata, H. (2005), "A fast boundary element method for the analysis of fiber-reinforced composites based on a rigid-inclusion model”, Journal of Applied Mechanics, Vol. 72, pp. 115-28. 
Ma, F., Chatterjee, J., Henry, D.P. and Banerjee, P.K. (2008), "Transient heat conduction analysis of 3D solids with fiber inclusions using the boundary element method", International Journal for Numerical Methods in Engineering, Vol. 73, pp. 1113-36.

Mitic, P. and Rashed, Y.F. (2004), "Convergence and stability of the method of meshless fundamental solutions using an array of randomly distributed sources", Engineering Analysis with Boundary Elements, Vol. 28, pp. 143-53.

Qin, Q.H. (1993), "Nonlinear analysis of Reissner plates on an elastic foundation by the BEM", International Journal of Solids and Structures, Vol. 30, pp. 3101-11.

Qin, Q.H. (1994), "Hybrid Trefftz finite element approach for plate bending on an elastic foundation", Applied Mathematical Modelling, Vol. 18, pp. 334-9.

Qin, Q.H. (1995), "Hybrid Trefftz finite element method for Reissner plates on an elastic foundation", Computer Methods in Applied Mechanics and Engineering, Vol. 122, pp. 379-92.

Qin, Q.H. (2000), The Trefftz Finite and Boundary Element Method, WIT Press, Southampton.

Qin, Q.H. (2003), "Solving anti-plane problems of piezoelectric materials by the Trefftz finite element approach”, Computational Mechanics, Vol. 31, pp. 461-8.

Qin, Q.H. (2004), "Dual variational formulation for Trefftz finite element method of elastic materials", Mechanics Research Communications, Vol. 31, pp. 321-30.

Qin, Q.H. and Wang, H. (2008), Matlab and C Programming for Trefftz Finite Element Methods, CRC Press, New York, NY.

Wang, H. and Qin, Q.H. (2007), "Some problems with the method of fundamental solution using radial basis functions", Acta Mechanica Solida Sinica, Vol. 20, pp. 21-9.

Wang, H. and Qin, Q.H. (2008), "Meshless approach for thermo-mechanical analysis of functionally graded materials", Engineering Analysis with Boundary Elements, Vol. 32, pp. 704-12.

Wang, H. and Qin, Q.H. (2010), "Fundamental-solution-based finite element model for plane orthotropic elastic bodies", European Journal of Mechanics A/Solids, Vol. 29, pp. 801-9.

Wang, H and Yao, Z.H. (2007), "Large scale analysis of mechanical properties in 3-D fiber-reinforced composites using a new fast multipole boundary element method", Tsinghua Science \& Technology, Vol. 12, pp. 554-61.

Wang, H. and Qin, Q.H. (2009), "Hybrid FEM with fundamental solutions as trial functions for heat conduction simulation”, Acta Mechanica Solida Sinica, Vol. 22, pp. 487-98.

Wang, H., Qin, Q.H. and Kang, Y.L. (2006), "A meshless model for transient heat conduction in functionally graded materials”, Computational Mechanics, Vol. 38, pp. 51-60.

Young, D.L., Jane, S.J., Fan, C.M., Murugesan, K. and Tsai, C.C. (2006), "The method of fundamental solutions for 2D and 3D Stokes problems", Journal of Computational Physics, Vol. 211, pp. 1-8.

\section{Corresponding author}

Qinghua Qin can be contacted at: qinghua.qin@anu.edu.au

\section{Fiber-reinforced composites}

1097 